\title{
Subacute thyroiditis during the COVID-19 pandemic: a prospective study
}

\author{
A. B. Bahçecioğlu' ${ }^{10} \cdot$ Z. C. Karahan ${ }^{2} \cdot$ B. İ. Aydoğan ${ }^{3} \cdot$ İ. A. Kalkan ${ }^{4} \cdot$ A. Azap ${ }^{4} \cdot$ M. F. Erdoğan ${ }^{1}$
}

Received: 17 October 2021 / Accepted: 29 November 2021 / Published online: 13 January 2022

(C) Italian Society of Endocrinology (SIE) 2021

\begin{abstract}
Purpose Subacute thyroiditis(SAT) is a destructive thyroiditis associated with viral infections. Several SAT cases associated with SARS-CoV-2 infection/vaccination were recently reported. We aimed to evaluate prospectively all cases applied to our tertiary center and their relationship with SARS-CoV-2 during 16 months of the pandemic. Cases during similar prepandemic period were recorded for numeric comparison.

Methods Prospective study took place between March 2020 and July 2021. SAT was diagnosed by classical criteria. Swabs for SARS-CoV-2 and a wide respiratory viral panel (RV-PCR) were taken. Previous COVID-19 was assessed by SARSCoV-2 IgM\&IgG levels. Study group was divided into three as: CoV-SAT, patients who had or still have COVID-19, VacSAT, patients diagnosed within three months after SARS-CoV-2 vaccination and NonCoV-SAT, those not associated with COVID-19 or vaccination.

Results Out of 64 patients, $18.8 \%(n=12)$ was classified as CoV-SAT, 9.3\% $(n=6)$ as Vac-SAT and 71.9\% as $(n=46)$ NonCoV-SAT. SARS-CoV-2 RT-PCR tests on the diagnosis of SAT were negative in all, but two patients tested positive five days later, in second testing, performed upon clinical necessity. CoV-SAT and NonCoV-SAT groups were similar in terms of clinical, laboratory, and treatment characteristics. However, symptoms were milder and treatment was easier in Vac-SAT group $(p=0.006)$.

Conclusions Total number of SAT cases during the pandemic period was comparable to pre-pandemic period. However, a considerable rate of SARS-CoV-2 exposure in SAT patients was established. COVID-19 presented with SAT, as the first manifestation in three cases. Vaccine-related cases developed in a shorter time period, clinical presentation was milder, and only a few required corticosteroids.
\end{abstract}

Keywords SARS-CoV-2 $\cdot$ Subacute thyroiditis $\cdot$ COVID-19 $\cdot$ Pandemic $\cdot$ Vaccine

A. B. Bahçecioğlu

begumbahceci@hotmail.com

Z. C. Karahan

cerenkarahan@hotmail.com

B. İ. Aydoğan

imgehalici@gmail.com

İ. A. Kalkan

iremakd@yahoo.com

A. Azap

azap@medicine.ankara.edu.tr

M. F. Erdoğan

murat.erdogan@temd.org.tr
1 Department of Endocrinology and Metabolism, School of Medicine, Ankara University, Ankara, Turkey

2 Department of Medical Microbiology, School of Medicine, Ankara University, Ankara, Turkey

3 Department of Endocrinology and Metabolism, Güven Hospital, Ankara, Turkey

4 Department of Clinical Microbiology and Infectious Diseases, School of Medicine, Ankara University, Ankara, Turkey 


\section{Introductions}

Severe acute respiratory syndrome coronavirus 2 (SARS$\mathrm{CoV}-2$ ) has infected millions of people all over the world [1]. SARS-CoV-2 uses human angiotensin converting enzyme 2 (ACE2) for cell entry and transmembrane serine protease 2 receptor (TMPRSS2) for S- protein priming [2]. ACE2 and TMPRSS2 are expressed not only in the upper and lower respiratory tract but also in tissues, like thyroid, exocrine pancreas, intestinal tract, heart, proximal renal tubules, adipocytes, Sertoli and Leydig cells [3-6]. Possible mechanisms of virus-related injury are direct viral action or indirect damage through inflammatory and immunological pathways. 'Immunothrombosis', cooperation of immune and coagulation systems to block pathogens and 'endothelial dysfunction' are the main pathogenic mechanisms [7]. Vascular endothelial cells are infected by SARS-CoV-2, resulting in cellular damage and apoptosis leading to a prothrombotic state. Since COVID-19 is a hyperinflammatory state, autoimmunity may be triggered through inflammatory dysregulation [8]. Previous data propose COVID-19 initiated autoimmune diseases such as Graves' disease, antiphospholipid syndrome, autoimmune hemolytic anemia etc. [9].

Reports are accumulating in the literature, suggesting that the thyroid can also be affected by similar mechanisms $[10,11]$. Postmortem histopathological studies showed a high level of SARS-CoV-2 genome in the thyroid but no virus-related morphological changes [12]. On the contrary, one report showed follicle epithelial cell damage [13]. Similarly, damage has been shown in studies related to SARS-CoV, which has a very comparable genomic structure to SARS-CoV-2 [14]. In addition, our current knowledge accumulated since the beginning of the pandemic has shown that SARS-CoV-2 can cause short-term and reversible thyroid dysfunction [15].

Subacute thyroiditis (SAT) is a destructive thyroiditis reported to be associated by viral infections such as mumps virus, coxsackievirus, adenovirus, measles virus, Cytomegalovirus, influenza virus etc. and presented with self-limiting inflammation [16]. During the pandemic, several case reports have been published, suggestive of SAT associated with SARS-CoV-2. Brancatella et al. reported the first case of SAT related to SARS-CoV-2 infection [17]. Subsequently, four more cases of SAT developed 16-36 days after SARS-CoV-2 infection were published by the same authors [18] With these cases, a total of 30 cases were reported worldwide [10, 19-24]. Four of them were diagnosed with SAT simultaneously with the COVID-19, and 26 were five to 90 days later. Besides, twenty cases of SAT following SARS-CoV-2 vaccination have been described [25-34]. Subclinical and/or unreported cases of COVID-19 are also very common and may theoretically lead to SAT. Thus we aimed to systematically and prospectively follow all SAT cases and evaluate their relationship with SARS-CoV-2 infection/vaccination during first 16 months of pandemic. We investigated the clinical features and infectious, molecular and serological markers of all SAT cases diagnosed during this period.

\section{Methods}

\section{Study design and setting}

Patients diagnosed with SAT from a single tertiary care outpatient endocrinology clinic were prospectively included between March 2020 and July 2021. To compare prevalence of SAT in similar seasonal periods before and during the pandemic, Patients diagnosed with SAT between March 2018 and July 2019 were also recorded for retrospective review. Ethical approval has been obtained from ethical committee of Ankara University (Project number: İ8-505-20).

\section{Patients}

Patients with typical symptoms and findings of SAT were examined with thyroid function tests [sTSH, FT3, FT4], ESR, CRP levels and thyroid ultrasonography (TUS). Normal ranges in our lab for TSH, FT3, FT4, ESR and CRP levels are 0.38-5.33 mIU/mL, 3.99-6.71 pmol/L, $7-15.96 \mathrm{pmol} / \mathrm{L},<20 \mathrm{~mm} / \mathrm{h}$, and $<5 \mathrm{mg} / \mathrm{dl}$, respectively. Typical sonographic features of SAT included bilateral/ unilateral gland enlargement, focal ill-defined heterogeneous hypoechoic areas with decreased vascularity, and probe induced tenderness during examination. Patients older than 18 years who were diagnosed with SAT having typical symptoms and findings such as neck pain, fever, tenderness on palpation, elevated ESR/CRP serum levels, and typical sonographic findings were included.

Patients having an immunodeficiency that will affect serum immunoglobulin (Ig) levels such as common variable immune deficiency or receiving chemotherapy for cancer, rituximab for a rheumatologic disease, a supraphysiological dose of corticosteroids $(\geq 7.5 \mathrm{mg} /$ day of prednisolone) in the last three weeks before the blood sample is taken were excluded. As well as patients who were vaccinated for SARS-CoV-2 more than three months before SAT were excluded, since these are unlikely to be related to vaccination.

NSAIDs were preferred for patients with mild symptoms, partial gland involvement and/or to those cases with self-improvement history. Whereas corticosteroids were 
preferred for those with prominent symptoms, bilateral, diffuse involvement and/or those who did not respond to NSAIDs.

\section{Study protocol}

After inclusion, oro-nasopharyngeal swabs for SARS-CoV-2 and respiratory viral panel PCR (RV-PCR) were taken in symptomatic period. To assess SARS-CoV-2 IgM\&IgG antibody $(\mathrm{Ab})$ levels, blood samples were taken at least two weeks after the onset of symptoms, to allow sufficient time for antibody production. Serum was separated and stored at $-80{ }^{\circ} \mathrm{C}$ until serological testing.

The clinical spectrum of COVID-19 is classified according to the NIH, Coronavirus Disease 2019 (COVID-19) Treatment Guidelines as; asymptomatic, mild, moderate, severe and critical illness [35].

After assessment, patients were divided into three groups; patients have had or still have COVID-19 (CoVSAT), patients who were diagnosed with SAT within three months after SARS-CoV-2 vaccination (Vac-SAT), patients neither had COVID-19 nor with vaccinated for SARS-CoV-2 (NonCoV-SAT).

\section{Assays}

\section{Molecular analysis}

For RV-PCR analysis, viral RNA/DNA was extracted with the EZ1Virus Mini Kit using the EZ1 Advanced XL Platform (Qiagen ${ }^{\circledR}$, USA). Presence of respiratory pathogens other than SARS-CoV-2 were evaluated by multiplex PCR method using the Fast Track Diagnostics Respiratory Pathogens 21 Kit (FTD21, Fast Tract Diagnostics ${ }^{\circledR}$, Luxembourg) on the Rotorgene Q 5plex HRM System (Qiagen ${ }^{\circledR}$, USA). The kit contains specific primer-probes to detect common respiratory viruses including influenza viruses $A, A(H 1 N 1)$, and $\mathrm{B}$, parainfluenza viruses (PIVs) $1-4$, human rhinovirus (hRV), respiratory syncytial viruses (RSV) A/B, human metapneumoviruses A/B (hMPV), human coronaviruses (hCoVs) OC43, 229E, NL63, and HKU1, human adenovirus (ADV), enterovirus (EV), human parechovirus and human bocavirus (hBOV).

RT-PCR detection of SARS-CoV-2 was performed using one of the three commercial kits which were approved for detection of SARS-CoV-2 by the Ministry of Health of Turkey during the study period: Biospeedy SARS-CoV-2 Double Gene RT-qPCR (Bioeksen ${ }^{\circledR}$, Turkey), Diagnovital HS SARS-CoV-2 RT-PCR (RTA Laboratories ${ }^{\circledR}$, Turkey) or Coronex-COVID 19 (DS Bio and Nano Technology ${ }^{\circledR}$, Turkey).

\section{Serological testing}

IgM and IgG antibody levels were assessed via chemiluminescent microparticle immunoassay (CMIA) in the same session. Abbott ${ }^{\circledR}$ SARS-CoV-2 IgG II Quant assay was used for the quantitative detection of 'Spike IgG' antibodies, result $\geq 50.0 \mathrm{AU} / \mathrm{ml}$ were reported as positive. Abbott ${ }^{\circledR}$ SARS-CoV-2, IgM assay was used for the qualitative detection of 'Spike IgM' antibodies.

\section{Statistical analysis}

Statistical analysis was performed using SPSS version 22.0. Descriptive statistics were given as counts and percentages for categorical variables; interquartile ranges and median were given for non-parametric continuous variables; standard deviation and mean for parametric continuous variables. Chi square test was performed for categorical data. Kruskal-Wallis test was used to compare three groups on continuous variables. Mann Whitney $U$ test is used for posthoc analysis of Kruskal Wallis test. A $p$ value of less than 0.05 was considered statistically significant.

\section{Results}

\section{Characteristics of the study group}

80 patients were diagnosed with SAT between March 2020 and July 2021. After assessing the eligibility criteria, 64 patients constituted the study group. A total of 16 patients were excluded; two for previous SARS-CoV-2 vaccination, five for corticosteroid treatment, nine for not giving consent to participate. Since four patients opposed swab samples, they were evaluated only by serological testing. 60 patients were evaluated with both serological and molecular testing.

Mean age of study group was $45.5 \pm 11.3$ years, $64 \%$ $(n=41)$ were female. Neck pain was the most common symptom $(n=62,97 \%)$ followed by asthenia $(n=19,30 \%)$, weight loss $(n=14,22 \%)$ and fever $(n=15,23 \%)$. Mean ESR of whole sample was $51.7 \pm 29.5 \mathrm{~mm} / \mathrm{h}$. Median CRP level was 47.4 (IQR =73, min-max: 1.3-247) $\mathrm{mg} / \mathrm{L}$. Median TSH level was 0.015 (IQR $=0.04$, $\min -\max : 0.01-3.74)$, FT3 was $7 \mathrm{pmol} / \mathrm{L}(\mathrm{IQR}=2.75$, min-max: $3.67-19.5)$, FT4 was $24.8 \mathrm{pmol} / \mathrm{L}(\mathrm{IQR}=14.7$, min-max: $10.04-79)$.

In TUS, $86 \%(n=55)$ of the patients had bilateral and $9 \%(n=6)$ had unilateral involvement. In 5\% $(n=3)$ of the patients, unilateral involvement progressed to bilateral during the follow-up.

$28 \%(n=18)$ of patients were treated with NSAIDs, $53 \%$ $(n=34)$ patients required glucocorticoids. Treatment were started with NSAIDs and switched to glucocorticoids due to unresponsiveness in $13 \%(n=8)$ of the patients. Since the 
symptoms were mild at the time of diagnosis, no treatment was given in two patients. The median initial methylprednisolone dose was $24 \mathrm{mg}$ and was tapered off at a median time of $6.5 \pm 1.8$ weeks.

Both Anti-SARS-CoV-2 IgM and IgG antibodies were detected in seven $(11 \%)$ patients, all of whom had a history of COVID-19 in the previous (3-36) weeks. In eight patients (13\%), only Anti SARS-CoV-2 IgG antibodies were positive, three had a history of COVID-19 6-12 weeks ago, and five were vaccinated.

SARS-CoV-2 RT-PCR tests performed on the day SAT was diagnosed were negative in all patients, although two were reported positive on repeated performed in five days (see below). In the RV-PCR test panel, viral genome of a respiratory pathogen was detected in nine (14\%) patients;
Four patients were positive for $\mathrm{hRV}$, and one each was positive for EV, hCoV-HKU1, hCoV-NL63, hCoV-OC43 and AdV. One of these patients was in the Vac-SAT group and the others were in the NonCoV-SAT.

In the retrospective review, 81 patients were diagnosed and treated for SAT between March 2018 and July 2019 in our outpatient clinic (i.e., similar interval and seasonal periods before pandemic).

\section{Comparison of the three groups}

Descriptive and clinical characteristics of the participants of the study group as Cov-SAT, Vac-SAT, NonCoV-SAT are presented in Table 1. There was no difference between the

Table 1 Descriptive and clinical characteristics of the participants $(N=64)$

\begin{tabular}{|c|c|c|c|c|c|c|}
\hline & $\begin{array}{l}\text { CoV-SAT } \\
N=12(18.8 \%)\end{array}$ & $\begin{array}{l}\text { Vac-SAT } \\
N=6(9.3 \%)\end{array}$ & $\begin{array}{l}\text { NonCoV-SAT } \\
N=46(71.9 \%)\end{array}$ & $\chi^{2}$ & $\mathrm{df}$ & $p$ \\
\hline Gender, Female & $5(42)$ & $75(83)$ & $31(67)$ & 3.80 & 2 & 0.149 \\
\hline Fever & $3(25)$ & $2(33)$ & $10(22)$ & 0.418 & 2 & 0.812 \\
\hline Neck pain & $12(100)^{\mathrm{ab}}$ & $4(67)^{\mathrm{a}}$ & $46(100)^{\mathrm{b}}$ & 19.957 & 2 & $<0.001$ \\
\hline Asthenia & $2(17)$ & $4(67)$ & $11(24)$ & 5.715 & 2 & 0.057 \\
\hline Sweating & $3(25)$ & $1(17)$ & $5(11)$ & 1.610 & 2 & 0.447 \\
\hline Weight loss & $2(17)^{\mathrm{a}}$ & $4(67)^{\mathrm{b}}$ & $8(17)^{\mathrm{a}}$ & 7.775 & 2 & 0.020 \\
\hline USG & & & & 2.594 & 4 & \\
\hline Unilateral & $2(17)$ & $0(0)$ & $4(9)$ & & & \\
\hline Bilateral & $10(83)$ & $6(100)$ & $39(85)$ & & & \\
\hline Uni to Bilateral* & $0(0)$ & $0(0)$ & $3(6)$ & & & \\
\hline SARS-CoV-2 IgG +** & $10(100)$ & $5(83)$ & $0(0)$ & NA & & \\
\hline SARS-CoV-2 IgM + ** & $7(70)$ & $0(0)$ & $0(0)$ & NA & & \\
\hline Medication & & & & 6.455 & 4 & 0.168 \\
\hline NSAID & $2(18)$ & $4(67)$ & $12(28)$ & & & \\
\hline Corticosteroid & $6(55)$ & $2(33)$ & $26(60)$ & & & \\
\hline \multirow[t]{2}{*}{ NSAID + Corticosteroid $* * *$} & $3(27)$ & $0(0)$ & $5(12)$ & & & \\
\hline & Median (IQR) & Median (IQR) & Median (IQR) & $\chi^{2} \#$ & $\mathrm{df}$ & $p$ \\
\hline Age & $49(13)$ & $56(42)$ & $44.5(10)$ & 2.894 & 2 & 0.235 \\
\hline $\operatorname{ESR}(<20 \mathrm{~mm} / \mathrm{h})$ & $47.5(21)$ & $53.5(39)$ & $50.35(36)$ & 0.929 & 2 & 0.629 \\
\hline $\mathrm{CRP}(<5 \mathrm{mg} / \mathrm{dl})$ & $49.2(40.4)$ & $32.5(75)$ & $47.5(90.8)$ & 0.710 & 2 & 0.701 \\
\hline TSH (0.38-5.33 mIU/mL) & $0.015(0.04)^{\mathrm{a}}$ & $0.01(0)^{\mathrm{b}}$ & $0.02(0.11)^{\mathrm{a}}$ & 13.546 & 2 & 0.001 \\
\hline FT4 (7-15.96 pmol/L) & $27.9(8.66)$ & $37.2(46.7)$ & $22.42(16.46)$ & 5.285 & 2 & 0.071 \\
\hline FT3 (3.99-6.71 pmol/L) & $8.1(3.26)$ & $9.33(12)$ & $6.65(2.59)$ & 3.292 & 2 & 0.193 \\
\hline Duration of treatment (weeks) & $6(4)^{\mathrm{ab}}$ & $4.5(1)^{\mathrm{a}}$ & $7(2)^{b}$ & 10.208 & 2 & 0.006 \\
\hline
\end{tabular}

$\chi^{2}$ : Chi square test, ${ }^{\#}$ Kruskal Wallis test, $d f$ Degree of freedom, $I Q R$ interquartile range, $U S G$ Ultrasonography,

NSAID Non-steroidal Anti-inflammatory Drugs, CRP C-Reactive Protein

*The patient whose thyroid involvement started unilaterally and became bilateral in the follow-up

**Two cases with concomitant SARS-CoV-2 infection were excluded

***Patients who were started treatment with NSAIDs and switched to glucocorticoids due to unresponsiveness

NA: Not applicable

ab,a,b: Different symbols indicate that the values in the row are significantly different from each other (corrected $p<0.05$ ) 
groups in terms of age, gender, frequency of fever, asthenia and sweating, ESR, CRP level and TUS findings (Table 1).

Neck pain was significantly less frequent in Vac-SAT compared to NonCoV-SAT. TSH level was significantly lower in Vac-SAT when compared to the other groups. Weight loss was significantly more frequent in Vac-SAT compared to others (Table 1). The median duration of treatment was shorter in Vac-SAT compared to NonCoV-SAT. The percentage of patients treated with NSAIDs in VacSAT was higher than other groups, but the difference was not statistically significant (Table 1). Median time between COVID-19 and the onset of SAT symptoms in CoV-SAT was 10 weeks (IQR $=15$, min-max: $3-24)$ and 4 weeks $(\mathrm{IQR}=9.5$, min-max: $1-12)$ in Vac-SAT.

\section{Clinical features of COVID-19 associated SATs (CoV-SAT)}

Nine patients who developed SAT after COVID-19 and three patients who were simultaneously diagnosed with SAT (patients 10-11-12) were classified as CoV-SAT and are presented in Table 2. Eight of these 12 patients had a history of mild COVID-19, one had asymptomatic COVID-19 assessed by antibodies (Patient 9). Two patients whose SARS-CoV-2 RT-PCR tests on the day of SAT diagnosis were negative, however, new symptoms appeared (fever in one, cough in one) several days after the initiation of SAT treatment. Thus RT-PCR testing was repeated, and reported as 'positive' five days later (patients 10-11).

Patient 12 is a 48-year-old female, she was admitted to the hospital with neck pain, fever cough, dyspnea and diagnosed with SAT. SARS-CoV-2 PCR was negative on the day of the diagnosis. However, chest computed tomography was compatible with a typical mild COVID-19 pneumonia. She was started on $32 \mathrm{mg}$ methylprednisolone for SAT. Supportive care was given for moderate COVID-19, which resolved readily. However, in terms of SAT she was a challenging case with three recurrences i.e., two when methylprednisolone was reduced to $4 \mathrm{mg}$ and one soon after discontinuation of steroids. When methylprednisolone was reduced to $8 \mathrm{mg}$ at last recurrence, colchicine was added. In this way, steroid therapy could have been terminated. Colchicine maintained for additional four weeks and SAT treatment was successfully terminated. IgG and IgM antibodies measured nine months after the diagnosis were still positive (Table 2).

\section{Clinical features of SARS-CoV-2 vaccinated SATs (Vac-SAT)}

Six patients who had SAT within three months after SARSCoV-2 vaccination were classified as Vac-SAT and are presented in Table 3. Four patients had been vaccinated with two doses of Sinovac-CoronaVac ${ }^{\circledR}$ (inactivated whole virus vaccine), two with a single dose of Pfizer-BioNtech ${ }^{\circledR}$ (mRNA vaccine). IgG levels were positive in all but one who received a single dose of BioNtech four weeks ago (Table 3 ). This group generally had milder symptoms than other groups and four $(67 \%)$ of these patients were treated with NSAIDs (Table 1).

\section{Clinical features of SATs not associated SARS-CoV-2 infection/vaccine (NonCoV-SAT)}

$67 \%(n=31)$ of this group, which constituted $72 \%(n=46)$ of the whole sample, compramised female patients. All patients had neck pain. Median duration of treatment was 7 (IQR:2, min-max:1-12) weeks, and methylprednisolone were used in the treatment of $72 \%(n=31)$ of the patients.

\section{Discussion}

Subacute thyroiditis (SAT) is a destructive inflammatory thyroiditis probably caused by immune hyperactivation which is thought to be caused by viral and postviral causes [16]. While many respiratory viruses are involved in its etiology, SARS-CoV-2 was suggested to be a cause after the cases reported during COVID-19 pandemic [10, 17-24]. Thyroid damage can be directly related to viral cytotoxicity or an immune response mediated by molecular mimicry [ 4 , $13,36]$. Thyroid exhibits high mRNA expression of ACE2 and TMPRSS2 [4-6]. In a study examining the reaction of human monoclonal antibodies to SARS-CoV-2 proteins with tissue antigens, high homology between immunogenic epitopes of SARS-CoV-2 to human proteins, including thyroid peroxidase (TPO), was reported [36]. Current study is the first prospective study systematically followed SAT cases during the pandemic and examined the relationship with SARS-CoV-2 infection and vaccination.

Since the outbreak of the COVID-19 pandemic, when 64 of 80 patients who met the eligibility criteria were examined prospectively, we observed that $18.2 \%$ were associated with SARS-CoV-2. In an Italian case series, it was stated that four out of 10 SAT cases were associated with SARSCoV-2, a significant number, for the $1 \%$ exposure rate in the Italian population [18]. However, the study reflects the first 6-month period of the pandemic and is only a case series. In Turkey, with a population of 83 million, 5.5 million people were infected with SARS-CoV-2 during our study period [37]. Exposure rate was calculated to be $6.6 \%$ in the population and $18.2 \%$ of SAT patients were likely to be associated with SARS-CoV-2. This considerably high percentage strongly supports the view that SARS-CoV-2 may have been involved in the etiology of SAT. Also bearing in mind that there are many viral factors that can cause SAT, the rate of SARS-CoV-2 seems to be high among them. However, the 
Table 2 Clinical, biochemical and imaging features of patients who develop subacute thyroiditis (SAT) after COVID-19 (CoV-SAT)

\begin{tabular}{|c|c|c|c|c|c|c|c|c|}
\hline $\begin{array}{l}\text { Number of } \\
\text { Patient Age } \\
\text { and Gender }\end{array}$ & $\begin{array}{l}\text { Clinical } \\
\text { Spectrum }^{\#} \\
\text { and treatment } \\
\text { of COVID-19 }\end{array}$ & $\begin{array}{l}\text { Time from } \\
\text { COVID-19 to } \\
\text { SAT } \\
\text { (weeks) }\end{array}$ & $\begin{array}{l}\text { SAT symp- } \\
\text { toms }\end{array}$ & $\begin{array}{l}\text { Thyroid hormone } \\
\text { status } \\
\operatorname{ESR}(<20 \mathrm{~mm} / \mathrm{h}) \\
\operatorname{CRP}(<5 \mathrm{mg} / \mathrm{L})\end{array}$ & $\begin{array}{l}\text { Ultrasono- } \\
\text { graphic } \\
\text { involvement }\end{array}$ & $\begin{array}{l}\text { SARS- } \\
\text { CoV-2/ } \\
\text { respiratory } \\
\text { viral } \\
\text { panel (RV) } \\
\text { PCR }\end{array}$ & $\begin{array}{l}\text { SARS- } \\
\text { CoV-2 } \\
\text { IgG AU/mL } \\
(0-50) \\
\text { IgM Anti- } \\
\text { body }\end{array}$ & $\begin{array}{l}\text { Treatment } \\
\text { of SAT and } \\
\text { reccurence(s) } \\
\text { Dose of MPZ- } \\
\text { duration }\end{array}$ \\
\hline $\begin{array}{l}\text { Patient } 1 \\
48 \text { years } \\
\text { Female }\end{array}$ & $\begin{array}{l}\text { Outpatient, } \\
\text { Mild Ilness } \\
\text { Supportive } \\
\text { care, } \\
\text { Favipravir }\end{array}$ & 6 & $\begin{array}{l}\text { Neck pain } \\
\text { Asthenia }\end{array}$ & $\begin{array}{l}\text { Thyrotoxic } \\
49 \mathrm{~mm} / \mathrm{h} \\
54 \mathrm{mg} / \mathrm{L}\end{array}$ & Bilateral & $\begin{array}{l}\text { Negative } \\
\text { Negative }\end{array}$ & $\begin{array}{l}141 \mathrm{AU} / \mathrm{mL} \\
\text { Negative }\end{array}$ & $\begin{array}{l}24 \mathrm{mg}^{*}-6 \text { weeks } \\
\text { reccurence } \\
16 \mathrm{mg}^{*}-5 \text { weeks }\end{array}$ \\
\hline $\begin{array}{c}\text { Patient } 2 \\
40 \text { years, } \\
\text { Male }\end{array}$ & $\begin{array}{l}\text { Outpatient, } \\
\text { Mild Ilness } \\
\text { Supportive } \\
\text { care, } \\
\text { Favipravir }\end{array}$ & 13 & $\begin{array}{l}\text { Neck pain } \\
\text { Asthenia } \\
\text { Sweating } \\
\text { Palpitations }\end{array}$ & $\begin{array}{l}\text { Thyrotoxic } \\
17 \mathrm{~mm} / \mathrm{h} \\
49.6 \mathrm{mg} / \mathrm{L}\end{array}$ & Bilateral & $\begin{array}{l}\text { Negative } \\
\text { Negative }\end{array}$ & $\begin{array}{l}1175 \mathrm{AU} / \mathrm{mL} \\
\text { Positive }\end{array}$ & $24 \mathrm{mg} *-6$ weeks \\
\hline $\begin{array}{c}\text { Patient } 3 \\
40 \text { years, } \\
\text { Male }\end{array}$ & $\begin{array}{l}\text { Outpatient, } \\
\text { Mild Ilness } \\
\text { Supportive } \\
\text { care, } \\
\text { Favipravir }\end{array}$ & 12 & $\begin{array}{l}\text { Fever }\left(38^{\circ} \mathrm{C}\right) \\
\text { Neck pain } \\
\text { Asthenia } \\
\text { Weight loss } \\
\text { Sweating }\end{array}$ & $\begin{array}{l}\text { Thyrotoxic } \\
54 \mathrm{~mm} / \mathrm{h} \\
80 \mathrm{mg} / \mathrm{L}\end{array}$ & Unilateral & $\begin{array}{l}\text { Negative } \\
\text { Negative }\end{array}$ & $\begin{array}{l}811 \mathrm{AU} / \mathrm{mL} \\
\text { Negative }\end{array}$ & 32 mg*-6 weeks \\
\hline $\begin{array}{l}\text { Patient } 4 \\
56 \text { years, } \\
\text { Male }\end{array}$ & $\begin{array}{l}\text { Outpatient, } \\
\text { Mild Ilness } \\
\text { Supportive } \\
\text { care, } \\
\text { Favipravir }\end{array}$ & 8 & Neck pain & $\begin{array}{l}\text { Subclinical } \\
\text { hyperthyroid- } \\
\text { ism } \\
11 \mathrm{~mm} / \mathrm{h} \\
40 \mathrm{mg} / \mathrm{L}\end{array}$ & Bilateral & $\begin{array}{l}\text { Negative } \\
\text { Negative }\end{array}$ & $\begin{array}{l}1738 \mathrm{AU} / \mathrm{mL} \\
\text { Negative }\end{array}$ & $\begin{array}{l}\text { NSAID } \\
3 \text { weeks }\end{array}$ \\
\hline $\begin{array}{r}\text { Patient } 5 \\
54 \text { years, } \\
\text { Female }\end{array}$ & $\begin{array}{l}\text { Outpatient, } \\
\text { Mild Ilness } \\
\text { Supportive } \\
\text { care, } \\
\text { Favipravir }\end{array}$ & 3 & $\begin{array}{l}\text { Neck pain } \\
\text { Asthenia }\end{array}$ & $\begin{array}{l}\text { Thyrotoxic } \\
59 \mathrm{~mm} / \mathrm{h} \\
51 \mathrm{mg} / \mathrm{L}\end{array}$ & $\begin{array}{l}\text { Unilateral } \\
\text { (the left } \\
\text { lobe) }\end{array}$ & $\begin{array}{l}\text { Negative } \\
\text { Negative }\end{array}$ & $\begin{array}{l}1253 \mathrm{AU} / \mathrm{mL} \\
\text { Positive }\end{array}$ & $\begin{array}{l}\text { NSAID } \\
2 \text { weeks } \\
\text { unresponsive } \\
24 \mathrm{mg}^{*}-5 \text { weeks }\end{array}$ \\
\hline $\begin{array}{l}\text { Patient } 6 \\
52 \text { years, } \\
\text { Male }\end{array}$ & $\begin{array}{l}\text { Outpatient, } \\
\text { Mild Ilness } \\
\text { Supportive } \\
\text { care, } \\
\text { Favipravir }\end{array}$ & 7 & $\begin{array}{l}\text { Neck pain } \\
\quad \text { Asthenia } \\
\text { Sweating } \\
\text { Palpitations }\end{array}$ & $\begin{array}{l}\text { Thyrotoxic } \\
31 \mathrm{~mm} / \mathrm{h} \\
94.4 \mathrm{mg} / \mathrm{L}\end{array}$ & Bilateral & $\begin{array}{l}\text { Negative } \\
\text { Negative }\end{array}$ & $\begin{array}{l}3647 \mathrm{AU} / \mathrm{mL} \\
\text { Positive }\end{array}$ & $\begin{array}{l}\text { NSAID } \\
1 \text { week-unre- } \\
\text { sponsive } \\
16 \mathrm{mg}^{*} \text {-4 weeks }\end{array}$ \\
\hline $\begin{array}{l}\text { Patient } 7 \\
37 \text { years, } \\
\text { Male }\end{array}$ & $\begin{array}{l}\text { Outpatient, } \\
\text { Mild Ilness } \\
\text { Supportive } \\
\text { care, } \\
\text { Favipravir }\end{array}$ & 24 & Neck pain & $\begin{array}{l}\text { Thyrotoxic } \\
39 \mathrm{~mm} / \mathrm{h} \\
19.4 \mathrm{mg} / \mathrm{L}\end{array}$ & Bilateral & $\begin{array}{l}\text { Negative } \\
\text { Negative }\end{array}$ & $\begin{array}{l}167 \mathrm{AU} / \mathrm{mL} \\
\text { Positive }\end{array}$ & $\begin{array}{l}\text { NSAID } \\
4 \text { weeks }\end{array}$ \\
\hline $\begin{array}{l}\text { Patient } 8 \\
51 \text { years, } \\
\text { Male }\end{array}$ & $\begin{array}{l}\text { Outpatient, } \\
\text { Mild Ilness } \\
\text { Supportive } \\
\text { care, } \\
\text { Favipravir }\end{array}$ & 24 & $\begin{array}{l}\text { Neck pain } \\
\text { Sweating }\end{array}$ & $\begin{array}{l}\text { Subclinical } \\
\text { hyperthyroid- } \\
\text { ism } \\
35 \mathrm{~mm} / \mathrm{h} \\
48.7 \mathrm{mg} / \mathrm{L}\end{array}$ & Bilateral & $\begin{array}{l}\text { Negative } \\
\text { Negative }\end{array}$ & $\begin{array}{l}568 \mathrm{AU} / \mathrm{mL} \\
\text { Positive }\end{array}$ & $\begin{array}{l}\text { NSAID } \\
1 \text { week-unre- } \\
\text { sponsive } \\
32 \mathrm{mg} *-7 \text { weeks }\end{array}$ \\
\hline $\begin{array}{l}\text { Patient } 9 \\
36 \text { years, } \\
\text { Female }\end{array}$ & $\begin{array}{l}\text { Asympto- } \\
\text { matic } \\
\text { No treatment }\end{array}$ & N/A & $\begin{array}{l}\text { Neck pain } \\
\text { Weight loss }\end{array}$ & $\begin{array}{l}\text { Thyrotoxic } \\
71 \mathrm{~mm} / \mathrm{h} \\
22.5 \mathrm{mg} / \mathrm{L}\end{array}$ & $\begin{array}{l}\text { Unilateral } \\
\text { (the left } \\
\text { lobe) }\end{array}$ & $\begin{array}{l}\text { Negative } \\
\text { Negative }\end{array}$ & $\begin{array}{l}1176 \mathrm{AU} / \mathrm{mL} \\
\text { Positive }\end{array}$ & No treatment \\
\hline $\begin{array}{l}\text { Patient } 10 \\
53 \text { years, } \\
\text { Female }\end{array}$ & $\begin{array}{l}\text { Outpatient, } \\
\text { Mild Ilness } \\
\text { Supportive } \\
\text { care, } \\
\text { Favipravir }\end{array}$ & $\begin{array}{l}* * \\
\text { Concurrent }\end{array}$ & $\begin{array}{l}\text { Neck pain } \\
\text { Asthenia } \\
\text { Fever }\left(38{ }^{\circ} \mathrm{C}\right)\end{array}$ & $\begin{array}{l}\text { Subclinical } \\
\text { hyperthyroid- } \\
\text { ism } \\
46 \mathrm{~mm} / \mathrm{h} \\
7.6 \mathrm{mg} / \mathrm{L}\end{array}$ & Bilateral & $\begin{array}{l}\text { Negative } \\
\text { Negative }\end{array}$ & $\begin{array}{l}0.8 \mathrm{AU} / \mathrm{mL} \\
\text { Negative }\end{array}$ & 32 mg*-6 weeks \\
\hline $\begin{array}{l}\text { Patient } 11 \\
50 \text { years, } \\
\text { Male }\end{array}$ & $\begin{array}{l}\text { Outpatient, } \\
\text { Mild Ilness } \\
\text { Supportive } \\
\text { care, } \\
\text { Favipravir }\end{array}$ & $\begin{array}{l}* * \\
\text { Concurrent }\end{array}$ & $\begin{array}{l}\text { Neck pain } \\
\text { Sweating } \\
\text { Palpitations } \\
\text { Cough }\end{array}$ & $\begin{array}{l}\text { Thyrotoxic } \\
50 \mathrm{~mm} / \mathrm{h} \\
26 \mathrm{mg} / \mathrm{L}\end{array}$ & Bilateral & $\begin{array}{l}\text { Negative } \\
\text { Negative }\end{array}$ & Nonavailable & 16 mg*-4 weeks \\
\hline
\end{tabular}


Table 2 (continued)

\begin{tabular}{|c|c|c|c|c|c|c|c|c|}
\hline $\begin{array}{l}\text { Number of } \\
\text { Patient Age } \\
\text { and Gender }\end{array}$ & $\begin{array}{l}\text { Clinical } \\
\text { Spectrum }^{\#} \\
\text { and treatment } \\
\text { of COVID-19 }\end{array}$ & $\begin{array}{l}\text { Time from } \\
\text { COVID-19 to } \\
\text { SAT } \\
\text { (weeks) }\end{array}$ & $\begin{array}{l}\text { SAT symp- } \\
\text { toms }\end{array}$ & $\begin{array}{l}\text { Thyroid hormone } \\
\text { status } \\
\operatorname{ESR}(<20 \mathrm{~mm} / \mathrm{h}) \\
\mathrm{CRP}(<5 \mathrm{mg} / \mathrm{L})\end{array}$ & $\begin{array}{l}\text { Ultrasono- } \\
\text { graphic } \\
\text { involvement }\end{array}$ & $\begin{array}{l}\text { SARS- } \\
\text { CoV-2/ } \\
\text { respiratory } \\
\text { viral } \\
\text { panel (RV) } \\
\text { PCR }\end{array}$ & $\begin{array}{l}\text { SARS- } \\
\text { CoV-2 } \\
\text { IgG AU/mL } \\
(0-50) \\
\text { IgM Anti- } \\
\text { body }\end{array}$ & $\begin{array}{l}\text { Treatment } \\
\text { of SAT and } \\
\text { reccurence(s) } \\
\text { Dose of MPZ- } \\
\text { duration }\end{array}$ \\
\hline $\begin{array}{l}\text { Patient } 12 \\
48 \text { years, } \\
\text { Female }\end{array}$ & $\begin{array}{l}\text { Outpatient, } \\
\text { Moderate } \\
\text { Ilness } \\
\text { Supportive } \\
\text { care }\end{array}$ & Concurrent & $\begin{array}{l}\text { Neck pain } \\
\text { Fever }\left(38^{\circ} \mathrm{C}\right) \\
\text { Cough } \\
\text { Dispnea }\end{array}$ & $\begin{array}{l}\text { Thyrotoxic } \\
50 \mathrm{~mm} / \mathrm{h} \\
67 \mathrm{mg} / \mathrm{L}\end{array}$ & Bilateral & $\begin{array}{l}\text { Negative } \\
\text { Negative }\end{array}$ & $\begin{array}{l}\text { *** } \\
93.5 \mathrm{AU} / \mathrm{mL} \\
\text { Positive }\end{array}$ & $\begin{array}{l}32 \mathrm{mg}^{*}-6 \text { weeks } \\
\text { reccurence } \\
32 \mathrm{mg}{ }^{*}-8 \text { weeks } \\
\text { reccurence } \\
32 \mathrm{mg}{ }^{*}- \\
10 \text { weeks } \\
\text { reccurence } \\
32 \mathrm{mg}{ }^{*}- \\
12 \text { weeks } \\
+ \\
\text { Colchicine } 1 \mathrm{mg} \\
(9 \text { weeks) }\end{array}$ \\
\hline
\end{tabular}

*As the starting dose with gradually tapering schedule

**PCR was negative on the day of the diagnosis of SAT, and PCR repeated 5 days later due to the addition of new symptoms (fever/cough). Repeated PCR tests resulted as positive

***At the time of recurrences, 9 months after COVID and the initial diagnosis of subacute thyroiditis

\#COVID-19 Treatment Guidelines Panel. Coronavirus Disease 2019 (COVID-19) Treatment Guidelines. National Institutes of Health. Available at https://www.covid19treatmentguidelines.nih.gov/. (Accessed 21 July 2021). MPZ methylprednisolone

Table 3 Clinical, biochemical and imaging features of patients who develop subacute thyroiditis after SARS-CoV-2 vaccination (Vac-SAT)

\begin{tabular}{|c|c|c|c|c|c|c|c|c|}
\hline $\begin{array}{l}\text { Number of } \\
\text { Patient Age } \\
\text { and Gender }\end{array}$ & $\begin{array}{l}\text { Brand name } \\
\text { of vaccine }\end{array}$ & $\begin{array}{l}\text { Time from } \\
\text { vaccine to } \\
\text { SAT } \\
\text { (weeks) }\end{array}$ & $\begin{array}{l}\text { SAT } \\
\text { symptoms }\end{array}$ & $\begin{array}{l}\text { Thyroid } \\
\text { hormone } \\
\text { status } \\
\operatorname{ESR}(<20 \\
\mathrm{mm} / \mathrm{h}) \\
\mathrm{CRP}(<5 \mathrm{mg} / \mathrm{L})\end{array}$ & $\begin{array}{l}\text { Ultrasono- } \\
\text { graphic } \\
\text { involvement }\end{array}$ & $\begin{array}{l}\text { SARS- } \\
\text { CoV-2/ } \\
\text { respiratory } \\
\text { viral panel } \\
\text { (RV) PCR }\end{array}$ & $\begin{array}{l}\text { SARS-CoV-2 } \\
\text { IgG AU/mL } \\
(0-50) \\
\text { IgM Anti- } \\
\text { body }\end{array}$ & $\begin{array}{l}\text { Treatment } \\
\text { of SAT and } \\
\text { reccurence(s) } \\
\text { Dose of MPZ- } \\
\text { duration }\end{array}$ \\
\hline $\begin{array}{r}\text { Patient } 1 \\
82 \text { years, } \\
\text { Female }\end{array}$ & CoronaVac® & 2 & $\begin{array}{l}\text { Fever } \\
\quad\left(37.5^{\circ} \mathrm{C}\right) \\
\text { Tremor } \\
\text { Weight loss }\end{array}$ & $\begin{array}{l}\text { Thyrotoxic } \\
86 \mathrm{~mm} / \mathrm{h} \\
81 \mathrm{mg} / \mathrm{L}\end{array}$ & Bilateral & $\begin{array}{l}\text { Negative } \\
\text { Negative }\end{array}$ & $\begin{array}{l}456 \mathrm{AU} / \mathrm{mL} \\
\text { Negative }\end{array}$ & $\begin{array}{l}\text { NSAID } \\
4 \text { weeks } \\
\text { ß -blockers }\end{array}$ \\
\hline $\begin{array}{r}\text { Patient } 2 \\
79 \text { years, } \\
\text { Female }\end{array}$ & CoronaVac ${ }^{\circledR}$ & 1 & $\begin{array}{l}\text { Fever }\left(37^{\circ} \mathrm{C}\right) \\
\text { Tremor } \\
\text { Anorexia } \\
\text { Asthenia } \\
\text { Weight loss }\end{array}$ & $\begin{array}{l}\text { Thyrotoxic } \\
43 \mathrm{~mm} / \mathrm{h} \\
17.6 \mathrm{mg} / \mathrm{L}\end{array}$ & Bilateral & $\begin{array}{l}\text { Negative } \\
\text { Negative }\end{array}$ & $\begin{array}{l}1562 \mathrm{AU} / \mathrm{mL} \\
\text { Negative }\end{array}$ & $\begin{array}{l}\text { NSAID } \\
6 \text { weeks } \\
\beta \text {-blockers }\end{array}$ \\
\hline $\begin{array}{r}\text { Patient } 3 \\
41 \text { years, } \\
\text { Female }\end{array}$ & $\begin{array}{l}\text { Pfizer-BioN- } \\
\text { tech® }\end{array}$ & 4 & Neck pain & $\begin{array}{l}\text { Thyrotoxic } \\
5 \mathrm{~mm} / \mathrm{h} \\
1.4 \mathrm{mg} / \mathrm{L}\end{array}$ & $\begin{array}{l}\text { Unilateral } \\
\text { (left lobe) }\end{array}$ & $\begin{array}{l}\text { Negative } \\
\text { Negative }\end{array}$ & $\begin{array}{l}36.7 \mathrm{AU} / \mathrm{mL} \\
\text { Negative }\end{array}$ & $\begin{array}{l}\text { NSAID } \\
4 \text { weeks }\end{array}$ \\
\hline $\begin{array}{r}\text { Patient } 4 \\
28 \text { years, } \\
\text { Female }\end{array}$ & CoronaVac ${ }^{\circledR}$ & 11 & $\begin{array}{l}\text { Neck pain } \\
\text { Weight loss }\end{array}$ & $\begin{array}{l}\text { Thyrotoxic } \\
42 \mathrm{~mm} / \mathrm{h} \\
45 \mathrm{mg} / \mathrm{L}\end{array}$ & $\begin{array}{l}\text { Unilateral } \\
\text { (left lobe) }\end{array}$ & $\begin{array}{l}\text { Negative } \\
\text { Negative }\end{array}$ & $\begin{array}{l}528 \mathrm{AU} / \mathrm{mL} \\
\text { Negative }\end{array}$ & $\begin{array}{l}\text { NSAID } \\
4 \text { weeks }\end{array}$ \\
\hline $\begin{array}{r}\text { Patient } 5 \\
41 \text { years, } \\
\text { Female }\end{array}$ & $\begin{array}{l}\text { Pfizer- BioN- } \\
\text { tech }{ }^{\circledR}\end{array}$ & 4 & $\begin{array}{l}\text { Neck pain } \\
\text { Palpitations } \\
\text { Sweating }\end{array}$ & $\begin{array}{l}\text { Thyrotoxic } \\
67 \mathrm{~mm} / \mathrm{h} \\
20 \mathrm{mg} / \mathrm{L}\end{array}$ & Bilateral & $\begin{array}{l}\text { Negative } \\
\text { Enterovirus }\end{array}$ & $\begin{array}{l}9558 \mathrm{AU} / \mathrm{mL} \\
\text { Negative }\end{array}$ & $\begin{array}{l}24 \mathrm{mg}^{*} \text { - } \\
5 \text { weeks } \\
\text { ß -blockers }\end{array}$ \\
\hline $\begin{array}{c}\text { Patient } 6 \\
71 \text { years, } \\
\text { Male }\end{array}$ & CoronaVac® & 12 & $\begin{array}{l}\text { Neck pain } \\
\text { Asthenia } \\
\text { Weight loss }\end{array}$ & $\begin{array}{l}\text { Thyrotoxic } \\
64 \mathrm{~mm} / \mathrm{h} \\
111 \mathrm{mg} / \mathrm{L}\end{array}$ & Bilateral & $\begin{array}{l}\text { Negative } \\
\text { Negative }\end{array}$ & $\begin{array}{l}224 \mathrm{AU} / \mathrm{mL} \\
\text { Negative }\end{array}$ & $\begin{array}{l}16 \mathrm{mg}^{*}- \\
5 \text { weeks }\end{array}$ \\
\hline
\end{tabular}

*As the starting dose with gradually tapering schedule. MPZ methylprednisolone 
proportional decrease in infection rate with other respiratory virus, which are less contagious than SARS-CoV-2 and probably less widespread than usual due to precautions taken and the use of facial masks, social distancing, etc. may have attributed this relatively high rate [38].

Although some authors thought the incidence of SAT is higher than expected in the general population during the pandemic [39], a study comparing the frequency of SAT among patients admitted to their centers during the pandemic with previous years showed that there was no increase in incidence [40]. In another study examining SAT cases during 2016-2020, a comparable number of SAT patients per year was reported from a single center [41]. Likewise, the number of patients diagnosed with SAT among our out patients within 16 months of the pre-pandemic period was similar to during the first 16 months of pandemic. Although it is not possible to reach a definite conclusion, with the experience of a single center, we did not find an increased frequency of SAT during the COVID-19 pandemic period. However, as stated before, the overall decrease of exposure to other respiratory viruses, as well as anxiety in the public for hospital admission, may be responsible for the similar frequency during the pandemic.

SAT is generally reported in 3rd-5th decade, and 1.9-6 times more frequent in females [42]. Consistent with the literature, mean age of our study group was $45.5 \pm 11.3$ years, $64 \%$ were female. However, F/M ratio was $1 / 4$ in the CoVSAT group, which is not relevant to the reported cases [i.e., 21 of $30(70 \%)$ cases presented as COVID-19 related SAT are women] [10, 17-24]. Recently, in an Italian study which examined SAT cases during the pandemic, reported 18 SARS-CoV-2-related cases who were all females [41]. On the other hand, men are more susceptible to COVID-19 and have a worse prognosis than women [43]. Mechanisms underlying this gender difference are still not clear. Possible suggested causes are activity of $\mathrm{X}$-linked genes which modulate immune response, sex steroids, differences in the expression of ACE2 and TMPRSS2 [44, 45]. However, there are also in vitro studies that do not show a gender-specific difference in the expression of ACE2 and TMPRSS2 receptors in the thyroid gland $[3,5]$.

In a systematic review of SARS-CoV-2-associated SAT cases, it was commented that the clinical features did not differ from classical SAT [46]. Consistent with this interpretation, in our study, CoV-SAT and NonCoV-SAT groups were similar regarding clinical features, laboratory findings and treatment characteristics.

Among the COVID-19 related 30 case reports described in the literature, 5 were concomitant, and 25 were postCOVID-19 cases. While our study, COVID-19 and SAT diagnosis were concomitant, in three cases. Two are, to our knowledge, the first described cases presenting initially with SAT and developing other COVID-19-related symptoms within a few days. Although this may be due to false-negative result of the first tests, our suggestion is to perform a second PCR test upon development of additional symptoms such as fever, cough, and dyspnea under SAT treatment.

Previously in 25 post-COVID-19 SAT cases, the interval between COVID-19 and onset of SAT symptoms was defined as 5-90 days (median 20 days) [10, 17-22, 24]. Our cases had a median interval of 10 weeks (IQR $=12$, 3-24 weeks), which is longer but also compatible with the classical knowledge of the time between viral infection and SAT.

In the previously described Italian study, when SARSCoV-2 positive and negative SAT cases were compared, significantly higher frequency of hypothyroidism at 3 months and CRP levels was reported in SARS-CoV-2 positive cases [41]. We found no difference between the CoV-SAT and the NonCoV-SAT groups, in terms of clinical and laboratory characteristics. More comprehensive studies are needed since both studies had small samples with regard to SARSCoV-2 related SAT.

Recently, several SAT cases appearing 1-21 days (median seven days) after SARS-CoV-2 vaccination was reported [25-34]. Median period was four weeks (1-12 weeks) for our six cases in Vac-SAT group. It is hard to prove that these cases are vaccination related, as are the other twenty cases reported [25-34]. However, it has been shown that this period can be as long as eight weeks in SAT cases after influenza vaccination [47]. Post-vaccine SAT seems to occur, relatively earlier than post-infection SAT, and relatively mild cases which usually respond to NSAIDs. However, thyrotoxicosis was more severe. These differences suggest that infection and vaccine associated SAT may occur through different mechanisms. As discussed earlier in a Turkish study, adjuvants in vaccine may cause ASIA (Autoimmune/ Inflammatory disease induced by adjuvants syndrome) by triggering adverse immune reactions in genetically predisposed individuals, associated with endocrine autoimmunity and SAT [25, 48]. In our study, four were after inactivated vaccine (CoronaVac $\left.{ }^{\circledR}\right)$ and two were after mRNA vaccine $\left(\right.$ Pfizer-BioNtech ${ }^{\circledR}$ ). Inactivated whole virus vaccine has multiple antigenic foci where as the only antigen formed by the mRNA vaccine is the 'Spike protein' [49]. However, other probable etiological agents for SAT cannot be excluded in these patients and this data needs verification by other prospective studies, including large scale vaccine trials.

Colchicine is an anti-inflammatory agent used in rheumatological diseases. We added to treatment of patient-12 (Table 2) whose SAT recurred for three times in nine months and it facilitated to discontinuation steroid therapy. A case series study from China reported colchicine helped with steroid dose reduction and discontinuation in recurrent subacute thyroiditis [50]. Although the mechanism of action for colchicine in SAT treatment is not yet known, a 
double-blind, controlled, prospective studies are needed to reach clear evidence.

It has always been difficult to demonstrate the viral etiology in SAT, indirect evidence has been used since direct evidence such as isolating the virus from the thyroid or serological evidence that will fully explain the immunological mechanism is lacking. Likewise, it is not possible to prove that SARS-CoV-2 or vaccination is involved in the etiology. Although we screened respiratory viral pathogens at the diagnosis, it is possible to dispute other factors may play a role. Another limitation is a serological evaluation of viral agents of upper respiratory tract infection in the previous weeks is not possible.

To conclude, although we did not observe a significant increase in the total number of SAT cases during pandemic, a relatively high rate of SARS-CoV-2 exposure in SAT patients was found. COVID-19 presented with SAT, as the first manifestation in three cases. Moreover, clinical and laboratory features are similar in COVID-19 related and nonrelated SAT cases. Vaccine-related cases developed in a shorter time period, clinical presentation was milder with less pain and only a few patients required corticosteroids.

Author contributions All the authors of the manuscript fulfill the criteria for authorship. ABB: Conception and design of the work, collecting data, analysis and interpretation of data, and article writing. ZCK: Performing molecular and serological analyses in the laboratory and revising the article. BİA: Design of the work and revising the article. IAK: Collecting data, design of the work and revising the article. AA: Design of the work and revising the article. MFE: Conception and design of the work, finding funds, collecting data, article writing and revising the article.

Funding Funded partly by Society of Endocrinology and Metabolism of Turkey (SEMT).

Data availability The data are available on request from the authors.

\section{Declarations}

Conflict of interest None of the authors have any potential conflicts of interest associated with this research.

Ethical approval Ethical approval has been obtained from ethical committee of Ankara University (Project number: İ8-505-20).

Informed consent Written informed consent was obtained from all the patients prior to inclusion.

\section{References}

1. WHO Coronavirus (COVID-19) Dashboard- available at https:// covid19. who.int/?gclid=EAIaIQobChMI07OH66DH8QIVk Lh3Ch31RwnbEAAYASABEgIvVPD_BwE. (Accessed 20 July 2021)
2. Hoffmann M, Kleine-Weber H, Schroeder S, Krüger N, Herrler T, Erichsen S et al (2020) SARS-CoV-2 cell entry depends on ACE2 and TMPRSS2 and is blocked by a clinically proven protease inhibitor. Cell 181(2):271-80.e8

3. Lazartigues E, Qadir MMF, Mauvais-Jarvis F (2020) Endocrine significance of SARS-CoV-2's reliance on ACE2. Endocrinology 161(9):108

4. Rotondi M, Coperchini F, Ricci G, Denegri M, Croce L, Ngnitejeu ST et al (2021) Detection of SARS-COV-2 receptor ACE-2 mRNA in thyroid cells: a clue for COVID-19-related subacute thyroiditis. J Endocrinol Invest 44(5):1085-1090

5. Li MY, Li L, Zhang Y, Wang XS (2020) Expression of the SARS$\mathrm{CoV}-2$ cell receptor gene ACE2 in a wide variety of human tissues. Infect Dis Poverty 9(1):45

6. Coperchini F, Ricci G, Croce L, Denegri M, Ruggiero R, Villani L et al (2021) Modulation of ACE-2 mRNA by inflammatory cytokines in human thyroid cells: a pilot study. Endocrine 74(3):638-645

7. Bonaventura A, Vecchié A, Dagna L, Martinod K, Dixon DL, Van Tassell BW et al (2021) Endothelial dysfunction and immunothrombosis as key pathogenic mechanisms in COVID-19. Nat Rev Immunol 21(5):319-329

8. Caso F, Costa L, Ruscitti P, Navarini L, Del Puente A, Giacomelli $\mathrm{R}$ et al (2020) Could Sars-coronavirus-2 trigger autoimmune and/ or autoinflammatory mechanisms in genetically predisposed subjects? Autoimmun Rev 19(5):102524

9. Saad MA, Alfishawy M, Nassar M, Mohamed M, Esene IN, Elbendary A (2021) Covid-19 and autoimmune diseases: a systematic review of reported cases. Curr Rheumatol Rev 17(2):193-204

10. Ruggeri RM, Campennì A, Deandreis D, Siracusa M, Tozzoli R, Petranović Ovčariček P et al (2021) SARS-COV-2-related immune-inflammatory thyroid disorders: facts and perspectives. Expert Rev Clin Immunol:1-23.

11. Mateu-Salat M, Urgell E, Chico A (2020) SARS-COV-2 as a trigger for autoimmune disease: report of two cases of Graves' disease after COVID-19. J Endocrinol Invest 43(10):1527-1528

12. Poma AM, Bonuccelli D, Giannini R, Macerola E, Vignali P, Ugolini C et al (2021) COVID-19 autopsy cases: detection of virus in endocrine tissues. J Endocrinol Invest pp. 1-6.

13. Hanley B, Naresh KN, Roufosse C, Nicholson AG, Weir J, Cooke GS et al (2020) Histopathological findings and viral tropism in UK patients with severe fatal COVID-19: a post-mortem study. Lancet Microbe 1(6):e245-e253

14. Wei L, Sun S, Xu C-H, Zhang J, Xu Y, Zhu H et al (2007) Pathology of the thyroid in severe acute respiratory syndrome. Hum Pathol 38(1):95-102

15. Lisco G, De Tullio A, Jirillo E, Giagulli VA, De Pergola G, Guastamacchia E et al (2021) Thyroid and COVID-19: a review on pathophysiological, clinical and organizational aspects. J Endocrinol Invest 44(9):1801-1814

16. Desailloud R, Hober D (2009) Viruses and thyroiditis: an update. Virol J 6:5

17. Brancatella A, Ricci D, Viola N, Sgrò D, Santini F, Latrofa F (2020) Subacute thyroiditis after Sars-COV-2 infection. J Clin Endocrinol Metab 105(7):2367-2370

18. Brancatella A, Ricci D, Cappellani D, Viola N, Sgrò D, Santini F et al (2020) Is Subacute thyroiditis an underestimated manifestation of SARS-CoV-2 Infection? Insights from a case series. J Clin Endocrinol Metab 105(10):e3742-e3746

19. Mehmood MA, Bapna M, Arshad M (2020) A case of postCOVID-19 subacute thyroiditis. Cureus 12(12):e12301

20. Abreu R, Miguel R, Saieg M (2021) Subacute (De Quervain) thyroiditis during the COVID-19 pandemic. Cancer Cytopathol.

21. de la Higuera L-F, Perdomo CM, Galofré JC (2021) Subacute thyroiditis following COVID-19 infection. Rev Clin Esp (Barc) 221(6):370-372 
22. Tjønnfjord E, Moe RB, Ghanima W, Aballi S (2021) Subacute thyroiditis after COVID-19. Tidsskr Nor Laegeforen 141(10).

23. Ippolito S, Dentali F, Tanda ML (2020) SARS-CoV-2: a potential trigger for subacute thyroiditis? Insights from a case report. J Endocrinol Invest 43(8):1171-1172

24. Asfuroglu Kalkan E, Ates I (2020) A case of subacute thyroiditis associated with Covid-19 infection. J Endocrinol Invest 43(8):1173-1174

25. İremli BG, Şendur SN, Ünlütürk U (2021) Three cases of subacute thyroiditis following SARS-CoV-2 vaccine: post-vaccination ASIA syndrome. J Clin Endocrinol Metab dgab373.

26. Franquemont S, Galvez J (2021) Subacute thyroiditis after mRNA vaccine for Covid-19. J Endocrine Soc 5(Suppl 1):A956-A957

27. Şahin Tekin M, Şaylısoy S, Yorulmaz G (2021) Subacute thyroiditis following COVID-19 vaccination in a 67-year-old male patient: a case report. Hum Vaccin Immunother pp.1-3.

28. Siolos A, Gartzonika K, Tigas S (2021) Thyroiditis following vaccination against COVID-19: report of two cases and review of the literature. Metabol Open 12:100136

29. Bornemann C, Woyk K, Bouter C (2021) Case report: two cases of subacute thyroiditis following SARS-CoV-2 vaccination. Front Med (Lausanne) 8:737142

30. Chatzi S, Karampela A, Spiliopoulou C, Boutzios G (2021) Subacute thyroiditis after SARS-CoV-2 vaccination: a report of two sisters and summary of the literature. Hormones (Athens) pp:1-3.

31. Patel KR, Cunnane ME, Deschler DG (2021) SARS-CoV-2 vaccine-induced subacute thyroiditis. Am J Otolaryngol 43(1):103211

32. Oyibo SO (2021) Subacute thyroiditis after receiving the adenovirus-vectored vaccine for coronavirus disease (COVID-19). Cureus 13(6): 16045

33. Lee KA, Kim YJ, Jin HY (2021) Thyrotoxicosis after COVID-19 vaccination: seven case reports and a literature review. Endocrine $12: 1-3$

34. Ratnayake GM, Dworakowska D, Grossman AB (2021) Can COVID-19 immunisation cause subacute thyroiditis? Clin Endocrinol (Oxf). https://doi.org/10.1111/cen.14555

35. COVID-19 Treatment Guidelines Panel. Coronavirus disease 2019 (COVID-19) treatment guidelines. National Institutes of Health. https://www.covid19treatmentguidelines.nih.gov/. (Accessed July 20,2021)

36. Vojdani A, Vojdani E, Kharrazian D (2020) Reaction of human monoclonal antibodies to SARS-CoV-2 proteins with tissue antigens: implications for autoimmune diseases. Front Immunol 11:617089

37. T.C. Sağlık Bakanlığ $\breve{l}_{1}$ COVID-19 Bilgilendirme Platformu-https:// covid19.saglik.gov.tr/. (Accessed 01 July 2021)

38. Olsen SJ, Winn AK, Budd AP, Prill MM, Steel J, Midgley CM et al (2021) Changes in influenza and other respiratory virus activity during the COVID-19 pandemic - united states, 2020 2021. MMWR Morb Mortal Wkly Rep 70(29):1013-1019

39. Muller I, Cannavaro D, Dazzi D, Covelli D, Mantovani G, Muscatello A et al (2020) SARS-CoV-2-related atypical thyroiditis. Lancet Diabetes Endocrinol 8(9):739-741

40. Pirola I, Gandossi E, Rotondi M, Marini F, Cristiano A, Chiovato $L$ et al (2021) Incidence of De Quervain's thyroiditis during the COVID-19 pandemic in an area heavily affected by Sars-CoV-2 infection. Endocrine 74(2):215-218

41. Brancatella A, Viola N, Rutigliano G, Sgrò D, Santini F, Latrofa F (2021) Subacute thyroiditis during the SARS-CoV-2 pandemic. J Endocr Soc 5(10):bvab130

42. Fatourechi V, Aniszewski JP, Fatourechi GZ, Atkinson EJ, Jacobsen SJ (2003) Clinical features and outcome of subacute thyroiditis in an incidence cohort: Olmsted County, Minnesota, study. J Clin Endocrinol Metab 88(5):2100-2105

43. Huang C, Wang Y, Li X, Ren L, Zhao J, Hu Y et al (2020) Clinical features of patients infected with 2019 novel coronavirus in Wuhan. China Lancet 395(10223):497-506

44. Peckham H, de Gruijter NM, Raine C, Radziszewska A, Ciurtin C, Wedderburn LR et al (2020) Male sex identified by global COVID-19 meta-analysis as a risk factor for death and ITU admission. Nat Commun 11(1):6317

45. Bouman A, Heineman MJ, Faas MM (2005) Sex hormones and the immune response in humans. Hum Reprod Update 11(4):411-423

46. Trimboli P, Cappelli C, Croce L, Scappaticcio L, Chiovato L, Rotondi M (2021) COVID-19-associated subacute thyroiditis: evidence-based data from a systematic review. Front Endocrinol (Lausanne) 12:707726

47. Altay FA, Güz G, Altay M (2016) Subacute thyroiditis following seasonal influenza vaccination. Hum Vaccin Immunother 12(4):1033-1034

48. Bragazzi NL, Hejly A, Watad A, Adawi M, Amital H, Shoenfeld Y (2020) ASIA syndrome and endocrine autoimmune disorders. Best Pract Res Clin Endocrinol Metab 34(1):101412

49. Heinz FX, Stiasny K (2021) Distinguishing features of current COVID-19 vaccines: knowns and unknowns of antigen presentation and modes of action. NPJ Vaccines 6(1):104

50. Tian Z, Su Y, Zhang M, Zhang X, Guan Q (2020) Successful management of recurrent subacute thyroiditis by adding colchicine to glucocorticoid treatment: a case series study. Horm Metab Res 52(10):712-717

Publisher's Note Springer Nature remains neutral with regard to jurisdictional claims in published maps and institutional affiliations. 